\title{
Approximation and Inapproximability Results for Maximum Clique of Disc Graphs in High Dimensions
}

\author{
Peyman Afshani ${ }^{a}$ and Hamed Hatami ${ }^{b}$ \\ Department of Computer Science \\ ${ }^{a}$ University of Waterloo \\ ${ }^{b}$ University of Toronto
}

\begin{abstract}
We prove algorithmic and hardness results for the problem of finding the largest set of a fixed diameter in the Euclidean space. In particular, we prove that if $A^{*}$ is the largest subset of diameter $r$ of $n$ points in the Euclidean space, then for every $\epsilon>0$ there exists a polynomial time algorithm that outputs a set $B$ of size at least $\left|A^{*}\right|$ and of diameter at most $r(\sqrt{2}+\epsilon)$. On the hardness side, roughly speaking, we show that unless $P=N P$ for every $\epsilon>0$ it is not possible to guarantee the diameter $r(\sqrt{4 / 3}-\epsilon)$ for $B$ even if the algorithm is allowed to output a set of size $\left(\frac{95}{94}-\epsilon\right)^{-1}\left|A^{*}\right|$.
\end{abstract}

Keywords: Approximation Algorithms; Computational Geometry; Disc Graphs; Diameter.

\section{Introduction}

The problem that we consider in this paper can be formulated as a clustering problem. These types of problems have been studied for quite long time and have many theoretical and practical applications in computer science [7]. A branch of clustering problems includes problems in which given a set of points the goal is to find a "cluster" (or clusters) with minimum size or maximum number of points. Typical examples of clusters include spheres, boxes, or any other shape of fixed complexity. Of course, the difficulty of the problem greatly depends on the definition of cluster. The clusters that we consider here are all the shapes of constant diameter. The diameter of a set $S$ is defined as

$$
\operatorname{diam}(S)=\sup _{x, y \in S}|x-y|,
$$

where $|x-y|$ is the Euclidean distance between the two points $x$ and $y$. Specifically, we consider the following problem:

Problem 1: Let $P$ be a set of $n$ points in $\mathbb{R}^{d}$ and $r>0$ be a real number. Find a subset $S \subseteq P$ of maximum size which satisfies $\operatorname{diam}(S) \leq r$. 
A clique is a graph in which every two vertices are adjacent. For a graph $G$, let $\omega(G)$ denote the size of the maximum clique in $G$, i.e. $\omega(G)$ is the maximum number of vertices of $G$ such that every two of them are adjacent. Determining $\omega(G)$ is called the maximum clique problem. A closely related topic is the notion of an independent set. An independent set in $G$ is a subset of vertices such that no two of them are adjacent. Let $\alpha(G)$ denote the size of the maximum independent set in $G$. Determining $\alpha(G)$ is called the maximum independent set problem. Denote by $G^{c}$ the complement of $G$, and note that $\omega(G)=\alpha\left(G^{c}\right)$. Thus, the maximum clique problem for $G$ is equivalent to the maximum independent set problem for $G^{c}$. For more on these topics we refer the reader to [17].

Problem 1 is equivalent to the maximum clique problem in disc graphs which are defined as follows. Given a point set $P \subset \mathbb{R}^{d}$ and a parameter $r$, a disc graph $G$ is defined by $V(G)=P$ and $x y \in E(G)$ if and only if $|x-y| \leq r$. There is a one to one correspondence between cliques in $G$ and sets of diameter at most $r$ in $P$.

In Problem 1 the diameter is fixed and our objective is to maximize the number of points. On the other hand, we can fix the number of points and ask for the minimum diameter:

Problem 2: Let $P$ be a set of $n$ points in $\mathbb{R}^{d}$ and $k>0$ be an integer. Find a subset $S \subseteq P$ of size $k$ with minimum diameter.

We show that both these problems are NP-hard when the dimension is sufficiently large, i.e., for some $d=\Theta(\log n)$. In fact, we prove a stronger result which shows that even certain approximations of these problems are impossible unless $\mathrm{P}=\mathrm{NP}$. These approximations are defined in the following way:

Definition. For $t, s \geq 1$, a $(t, s)$-approximation algorithm for Problem 1 is an algorithm that returns a set $S$ of size at least $O p t / t$ so that $\operatorname{diam}(S) \leq s r$, where $O p t$ is the size of the optimal answer to Problem 1.

Definition. For $t, s \geq 1$, a $(t, s)$-approximation algorithm for Problem 2 is an algorithm that returns a set $S$ of size at least $k / t$ so that $\operatorname{diam}(S) \leq s \times O p t$, where $O p t$ is the optimal answer to Problem 2.

These two problems are obtained by relaxing the size and diameter constraints of the output set. A simple observation shows that these two problems are equivalent.

Lemma 1 For $t, s \geq 1$, there exists a polynomial time $(t, s)$-approximation algorithm for Problem 1 if and only if there exists a polynomial time $(t, s)$-approximation algorithm for Problem 2.

Proof. Let $\mathcal{A}$ be a $(t, s)$-approximation algorithm for Problem 1. Consider an instance $(P, k)$ of Problem 2. For every pair of points $x, y \in P$, run $\mathcal{A}$ with parameter $r_{x, y}:=|x-y|$. We output the minimum $r_{x, y}$ for which the answer of $\mathcal{A}$ is of size at least $k / t$. Let $S_{o}$ be the optimal solution to the $(P, k)$ instance of Problem 2. At some point $\mathcal{A}$ is called with parameter $r:=\operatorname{diam}\left(S_{o}\right)$. Now the output of $\mathcal{A}$ is a set of size at least $\left|S_{o}\right| / t \geq k / t$ and with diameter at $\operatorname{most} r s=s \times \operatorname{diam}\left(S_{o}\right)$. 
To prove the other direction let $\mathcal{B}$ be a $(t, s)$-approximation algorithm for Problem 2 . Consider an instance $(P, r)$ for Problem 1. A $(t, s)$-approximation algorithm for Problem 1 can be obtained in a similar way by running $\mathcal{B}$ for every $k=1, \ldots, n$.

Since these two problems are equivalent we refer to both of them as the Diameter Approximation Problem.

Both Problems 1 and 2 are solvable in the 2-dimensional plane in polynomial time [2, 7, 8]. For Problem 2 the fastest known algorithm achieves the running time $O\left(n \log n+k^{2.5} n \log k\right)[8$. It is shown in [1] that in the 3-dimensional space there is a $\left(\frac{\pi}{\cos ^{-1} 1 / 3}, 1\right)$-approximation algorithm. Finally, when the dimension $d$ is a fixed constant, one can design a polynomial time approximation scheme achieving a $(1,1+\epsilon)$-approximation, for every $\epsilon>0$ [1]. It is also easy to see that there exists a trivial $(1,2)$-approximation algorithm for this problem in any dimension: a ball with radius $r$ about a point $x \in P$ containing the maximum number of points is a $(1,2)$-approximation for Problem 1 . Thus, it is interesting to study at which point the problem turns from polynomially solvable to NP-hard. We have the following result in this direction:

Theorem 2 For every $\epsilon>0$ there exists $d=\Theta(\log n)$, so that there is no polynomial time $\left(\frac{95}{94}-\right.$ $\epsilon, \sqrt{4 / 3}-\epsilon)$-approximation algorithm for the Diameter Approximation Problem in dimension $d$ unless $P=N P$.

We also improve upon the trivial $(1,2)$-approximation algorithm and obtain the following theorem:

Theorem 3 For every $\epsilon>0$ there is a polynomial time $(1, \sqrt{2}+\epsilon)$-approximation algorithm for the Diameter Approximation Problem in any dimension.

In Section 2.1 we prove Theorem 2. We use spectral properties to move from combinatorics of graphs to geometry of Euclidean space. This technique in combination with a hardness result regarding the maximum independent set problem in 3-regular graphs proves Theorem 2 . In Section 2.2 we prove Theorem 3 using simple geometric techniques. Finally, in the "Corollaries" subsection we observe that having Theorem 3 in hand, it is possible to move in the other direction. Corollaries 5 and 6 show that one can apply Theorem 3 to the geometric representation of the graph to approximate the maximum independent set problem for certain graphs.

\section{From Graphs to Euclidean Space}

In this section we prove Theorem 2. We show that unless $\mathrm{P}=\mathrm{NP}$, there is no $\left(\frac{95}{94}-\epsilon, \sqrt{4 / 3}-\epsilon\right)$ approximation algorithm for the Diameter Approximation Problem for every $\epsilon>0$. We use spectral techniques to show that a certain metric on the graph embeds isometrically into the Euclidean space. This type of reduction from geometry to graph theory via metric embedding has been successfully applied to various problems dealing with the so called $(1-2)$-metrics [10, 16]. Our proof of Theorem 2 relies on the following result:

Theorem 4 [5] For every $\epsilon>0$, unless $P=N P$, there is no polynomial time algorithm that approximates the maximum independent set problem in 3-regular graphs within a factor of $\frac{95}{94}-\epsilon$. 


\subsection{Proof of Theorem 2}

To prove the theorem we reduce the Diameter Approximation Problem to the maximum independent set problem in 3-regular graphs.

Consider a 3-regular graph $G$. Denote by $G^{c}$ the complement of $G$, and notice that cliques in $G^{c}$ correspond to independent sets in $G$. We begin by finding a lower bound for the minimum eigenvalue of the adjacency matrix $A_{G^{c}}$ of $G^{c}$. Denote by $\lambda_{1} \leq \ldots \leq \lambda_{n}$ the eigenvalues of $A_{G^{c}}$. Since $G^{c}$ is an $(n-4)$-regular graph, its Laplacian can be written as $L_{G^{c}}=(n-4) I-A_{G^{c}}$. Thus for a vector $v$, we have $A_{G^{c}} v=\lambda v$ if and only if $L_{G^{c}} v=(n-4-\lambda) v$. This shows that the eigenvalues of $L_{G^{c}}$ are $n-4-\lambda_{n} \leq \ldots \leq n-4-\lambda_{1}$. It is well-known [9] that the maximum eigenvalue of the Laplacian is bounded by $n$. So the minimum eigenvalue of $A_{G}$ is at least -4 . Define $Q:=A_{G^{c}}+(4+\gamma) I$ for $\gamma>0$ chosen sufficiently small. By the same argument that we used above to find the eigenvalues of $L_{G^{c}}$, one can show that the smallest eigenvalue of $Q$ is $\lambda_{1}+4+\gamma>0$ which means that $Q$ is a symmetric positive definite matrix. This implies that there exists a nonsingular matrix $U$, which can be found using elementary techniques, such that $Q=U^{t} U$ (see [11] page 285 for instance). Define a function $f:[n] \rightarrow \mathbb{R}^{n}$ by setting the value of $f(i)$ to be the $i$-th row of the matrix $U$. Note that

$$
|f(i)|^{2}=f(i) \cdot f(i)=Q_{i, i}=4+\gamma,
$$

and

$$
|f(i)-f(j)|^{2}=|f(i)|^{2}+|f(j)|^{2}-2 f(i) \cdot f(j)=8+2 \gamma-2 Q_{i, j} .
$$

Thus

$$
|f(i)-f(j)|= \begin{cases}\sqrt{6+2 \gamma} & A_{(i j)}^{c}=1 \\ \sqrt{8+2 \gamma} & A_{(i j)}^{c}=0\end{cases}
$$

Consider the vertex $v_{i}$ of $V\left(G^{c}\right)$ which corresponds to the $i$-th row and column of $A^{c}$. Map $v_{i}$ to the point $f(i)$ in Euclidean space $\mathbb{R}^{n}$. Let $P$ be the resulting point set in $\mathbb{R}^{n}$. The above properties imply that every vertex of $V\left(G^{c}\right)$ is mapped to a vector of magnitude 2 and the distance between two vertices is $\sqrt{6+2 \gamma}$, if there is an edge between them, and $\sqrt{8+2 \gamma}$ if not.

Using the Johnson-Lindenstrauss dimension reduction lemma [12, 13] there exists a dimension $d=O\left(\lambda^{-2} \log n\right)$, and a polynomial algorithm which maps $P$ into $\mathbb{R}^{d}$ such that the distance between any two points of $P$ changes by a factor of at most $1+\lambda / 2$. Let $g: V\left(G^{c}\right) \rightarrow \mathbb{R}^{d}$ be the corresponding map. Thus if we choose $\lambda$ and $\gamma$ small enough, for every two vertices $v_{i}, v_{j} \in V\left(G^{c}\right)$ the distance between $g(i)$ and $g(j)$ is at most $(1+\lambda) \sqrt{6}$ if they are connected in $G^{c}$ and at least $(1+\lambda)^{-1} \sqrt{8}$ if they are not connected in $G^{c}$. So for a set $S \subset V\left(G^{c}\right)$, its geometric representation will have diameter at most $(1+\lambda) \sqrt{6}$ if it is a clique but it will have diameter at least $(1+\lambda)^{-1} \sqrt{8}$ otherwise. By picking $\epsilon$ to be small enough and applying a $\left(\frac{95}{94}-\epsilon, \sqrt{4 / 3}-\epsilon\right)$-approximation algorithm to Problem 1 with $g\left(G^{c}\right)$ and $r=\sqrt{6}$ we can find a clique of size at least $1 /\left(\frac{95}{94}-\epsilon\right)$ times the size of the maximum clique in $G^{c}$. Theorem 4 shows that this is impossible unless $P=N P$. 


\subsection{Proof of Theorem 3}

In this section we prove Theorem 3 by applying simple geometric techniques. We follow the general ideas and techniques of [1, 2], borrowing and generalizing the main tool from [2]. The idea is to extend and generalize the trivial $(1,2)$-approximation. One way to interpret the $(1,2)$ approximation is to say that any set $A$ of diameter $r$ can be placed inside a ball of diameter $2 r$ centered at a point in $A$. To obtain a $(1, \sqrt{2}+\epsilon)$-approximation, we first show that any set of diameter $r$ can actually be placed inside a ball of diameter $(\sqrt{2}+\epsilon) r$, and then we produce a polynomial time algorithm to compute such a ball.

Let $A$ be the optimal answer to Problem 1. We start by proving that $A$ is inside a ball of diameter $(\sqrt{2}+\epsilon) r$. Let $B(P, t)$ denote a ball of radius $t$ centered at point $P$. At the beginning, $P_{1}$ is an arbitrary point of $A$ and thus we have $A \subset B\left(P_{1}, r\right)$. At the $i$-th step we assume $A \subset B\left(V_{i}, r_{i}\right)$ for a $V_{i} \in \mathbb{R}^{d}$ and some value $r_{i} \leq r$ to be determined later.

Let $P_{i+1}$ be the point of $A$ with maximum distance to $V_{i}$. This implies,

$$
A \subset B\left(V_{i}, r_{i}\right) \cap B\left(P_{i+1}, r\right) \cap B\left(V_{i},\left|V_{i}-P_{i+1}\right|\right)
$$

and since $\left|V_{i}-P_{i+1}\right| \leq r_{i}$, we have:

$$
A \subset B\left(P_{i+1}, r\right) \cap B\left(V_{i},\left|V_{i}-P_{i+1}\right|\right) .
$$

If $x=\left|V_{i}-P_{i+1}\right| \leq r \sqrt{2} / 2$ then the set of points is inside a ball of diameter $\sqrt{2} r$. So we assume $x>r \sqrt{2} / 2$.

Consider a point $L$ on the intersection of boundaries of the two balls $B\left(P_{i+1}, r\right)$ and $B\left(V_{i}, x\right)$ (Figure 1). Consider the plane passing through $L, P_{i+1}$ and $V_{i}$ and draw the line $L V_{i+1}$ perpendicular to the segment $P_{i+1} V_{i}$. A simple calculation proves that:

$$
\left|L-V_{i+1}\right|=r \sqrt{1-\frac{r^{2}}{4 x^{2}}} \leq r \sqrt{1-\frac{r^{2}}{4 r_{i}^{2}}}
$$

Define $r_{i+1}=r \sqrt{1-\frac{r^{2}}{4 r_{i}^{2}}}$. It can be also verified that if $x>r \sqrt{2} / 2$ then $\left|P_{i+1}-V_{i+1}\right|<$ $\left|V_{i+1}-L\right|$ and the ball $B\left(V_{i+1},\left|L-V_{i+1}\right|\right)$ will contain the intersection $B\left(P_{i+1}, r\right) \cap B\left(V_{i}, x\right)$. This implies $A \subset B\left(V_{i+1},\left|L-V_{i+1}\right|\right) \subset B\left(V_{i+1}, r_{i+1}\right)$. It is easy to check that the sequence $r_{1}, r_{2}, \cdots$ converges to $r \sqrt{2} / 2$. Thus given any $\epsilon>0$ it is possible to fix a constant $k$ (depending only on $\epsilon$ ) such that $r_{k}<r \sqrt{2} / 2+\epsilon$.

To obtain an algorithm from the discussion above, we only need to consider all different possible choices for $P_{1}, \ldots, P_{k}$. Discarding the invalid choices or choices that result in an invalid state, each choice for $P_{1}, \ldots, P_{k}$ leads to a ball with radius at most $r \sqrt{2} / 2+\epsilon$. Now the algorithm outputs the one which contains the maximum number of points. Since $k$ is a constant, the algorithm is polynomial.

\subsection{Corollaries}

The following corollaries can be obtained using techniques employed in the previous section. 


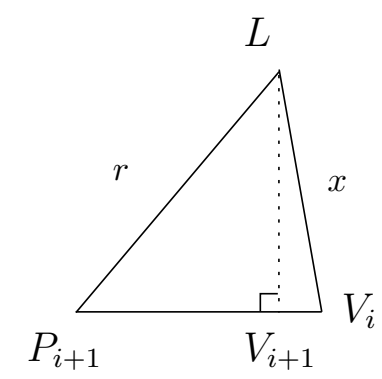

Figure 1: The position of $V_{i+1}$ with respect to the positions of $V_{i}, P_{i+1}$, and $L$.

Corollary 5 Fix $\delta>0$ and let $G$ be a graph such that there exists a mapping $f: V(G) \rightarrow \mathbb{R}^{n}$ satisfying $|f(u)-f(v)|>\sqrt{2}+\delta$ if $u v \in E(G)$, and $|f(u)-f(v)| \leq 1$ otherwise. Then it is possible to find the size of the maximum independent set of $G$ in polynomial time.

Proof. Let $V(G)=\left\{v_{1}, \ldots, v_{n}\right\}$. Suppose that the $i$-th row of a matrix $M$ be the vector $f\left(v_{i}\right)$. Let further $A=M M^{t}$. Clearly, $A$ is positive semi-definite, and $\left|f\left(v_{i}\right)-f\left(v_{j}\right)\right|^{2}=A_{i i}+A_{j j}-2 A_{i j}$. Thus finding the map $f$ reduces to finding a positive semi-definite matrix $A$ with

$$
A_{i i}+A_{j j}-2 A_{i j}>(\sqrt{2}+\delta)^{2} \quad \forall v_{i} v_{j} \in E(G),
$$

and

$$
A_{i i}+A_{j j}-2 A_{i j} \leq 1 \quad \forall i \neq j, v_{i} v_{j} \notin E(G) .
$$

As in ([14], Theorem 3.2) the ellipsoid algorithm can be invoked to find such a matrix $A$.

Then we apply the algorithm of Theorem 3 to $f(V(G))$ with the setting $r=1$ and $\epsilon=\delta / 2$. Let $I$ be an independent set of maximum size in $G$. Then the diameter of $f(I)$ is at most 1 because $|f(u)-f(v)| \leq 1$, if $u v \notin E(G)$. The algorithm given in the proof of Theorem 3 finds a set $P$ of diameter $\sqrt{2}+\delta / 2$ whose size is at least $|I|$. Since $|f(u)-f(v)|>\sqrt{2}+\delta$ when $u v \in E(G)$, we conclude that $f^{-1}(P)$ is an independent set. This completes the proof.

Corollary 6 Fix $\epsilon>0$ and let $G$ be a graph whose minimum eigenvalue is at least $-2+\epsilon$. It is possible to find the size of the maximum independent set of $G$ in polynomial time.

Proof. By the proof of Theorem 2 every such graph satisfies the condition of Corollary 5 .

\section{Concluding Remarks}

- We believe that Theorem 3 is "almost" sharp in the sense that the constant $\sqrt{4 / 3}-\epsilon$ in Theorem 2 can be improved to $\sqrt{2}-\epsilon$ becoming arbitrarily close to the $\sqrt{2}+\epsilon$ upper bound of Theorem 3 . 
- The main idea behind the proof of Theorem 3 was to introduce a polynomial time algorithm that given $n$ points computes a ball of diameter $(\sqrt{2}+\epsilon) r$ which contains the largest subset of the points that has diameter at most $r$. The fact that such a ball exists was already known, and in fact stronger results have been obtained using Helly-type arguments (we refer the reader to [6, 15] for the proofs and the description of the Helly-type theorems). However, the novel part of the proof was the algorithmic aspect, and showing that there exists a polynomial time algorithm which finds such a ball.

- There is already a body of work dedicated to characterization of all graphs with the smallest eigenvalue of at least -2 (see [3, 4]). These graphs have been characterized as "generalized line graphs" plus some finite set of exceptions. This characterization gives an alternative proof for Corollary 6 which uses a different polynomial time algorithm.

\section{Acknowledgements}

The authors would like to thank the anonymous referee for his/her helpful comments and suggestions that greatly helped us to remove some inaccuracies from the earlier version of this article.

\section{References}

[1] P. Afshani and T. Chan. Approximation algorithms for maximum cliques in 3D UnitDisk graphs. In Proceedings of the 17th Canadian Conference on Computational Geometry (CCCG'05), pages 19-22, 2005.

[2] A. Aggarwal, H. Imai, N. Katoh, and S. Suri. Finding $k$ points with minimum diameter and related problems. J. Algorithms, 12(1):38-56, 1991.

[3] F. C. Bussemaker and A. Neumaier. Exceptional graphs with smallest eigenvalue -2 and related problems. Math. Comp., 59(200):583-608, 1992.

[4] P. J. Cameron, J.-M. Goethals, J. J. Seidel, and E. E. Shult. Line graphs, root systems, and elliptic geometry. J. Algebra, 43(1):305-327, 1976.

[5] J. Chlebíková and M. Chlebík. Inapproximability results for bounded variants of optimization problems. Electronic Colloquium on Computational Complexity (ECCC), 10(026), 2003.

[6] L. Danzer, B. Grünbaum, and V. Klee. Helly's theorem and its relatives. In Proc. Sympos. Pure Math., Vol. VII, pages 101-180. Amer. Math. Soc., Providence, R.I., 1963.

[7] A. Datta, H.-P. Lenhof, C. Schwarz, and M. Smid. Static and dynamic algorithms for k-point clustering problems. J. Algorithms, 19(3):474-503, 1995. 
[8] D. Eppstein and J. Erickson. Iterated nearest neighbors and finding minimal polytopes. Discrete Comput. Geom., 11(3):321-350, 1994.

[9] C. Godsil and G. Royle. Algebraic graph theory, volume 207 of Graduate Texts in Mathematics. Springer-Verlag, New York, 2001.

[10] V. Guruswami and P. Indyk. Embeddings and non-approximability of geometric problems. In SODA '03: Proceedings of the Fourteenth Annual ACM-SIAM Symposium on Discrete Algorithms, pages 537-538, Philadelphia, PA, USA, 2003. Society for Industrial and Applied Mathematics.

[11] F. E. Hohn. Introduction to Linear Algebra. Macmillan, New York, 1972.

[12] P. Indyk and R. Motwani. Approximate nearest neighbors: towards removing the curse of dimensionality. In STOC '98: Proceedings of the Thirtieth Annual ACM Symposium on Theory of Computing, pages 604-613, New York, NY, USA, 1998. ACM Press.

[13] W. B. Johnson and J. Lindenstrauss. Extensions of Lipschitz mappings into a Hilbert space. In Conference in Modern Analysis and Probability (New Haven, Conn., 1982), volume 26 of Contemp. Math., pages 189-206. Amer. Math. Soc., Providence, RI, 1984.

[14] N. Linial, E. London, and Y. Rabinovich. The geometry of graphs and some of its algorithmic applications. Combinatorica, 15(2):215-245, 1995.

[15] J. Matoušek. Lectures on Discrete Geometry, volume 212 of Graduate Texts in Mathematics. Springer-Verlag, New York, 2002.

[16] L. Trevisan. When Hamming meets Euclid: the approximability of geometric TSP and MST (extended abstract). In STOC '9\%: Proceedings of the Twenty-Ninth Annual ACM Symposium on Theory of Computing, pages 21-29, New York, NY, USA, 1997. ACM Press.

[17] D. B. West. Introduction to graph theory. Prentice Hall Inc., Upper Saddle River, NJ, 1996. 